\title{
Sur quelques propriétés des isoclines dans un écoulement à deux dimensions ${ }^{(1)}$
}

\section{On some properties of isoclines in two-dimensional flow ${ }^{(1)}$}

\author{
PAR S. IRMAY
}

DIVISION OF HYDAAULIC ENGINEERING, ISRAEL, INSTITUTF OF TECHNOLOGY

\begin{abstract}
Les isoclines sont des lignes ayant la même inclinaison du vecteur de vitesse. Les isovels sont des lignes d'égale vitesse. Les isopièzes sont des lignes ayant la même hauteur piézométrique.

Dans un fluide non visqüeux et incompressible en écoulement potentiel permanent, les isoclines, dans un plán donné, sont normales anx lignes isopiëzes.

Cette propriété vaut dans le cas d'un écoulement rotatif dans un plan, mais ici les isopiezes ne sont pas identiques aux isovels. Dans les gaz, pour de faibles vitesses, les isoclines sont normales atu $x$ isbbares.

Ce théreme recoit de nombrenses applications: si l'on connait un groupe de lignes (isoclines ou isopièzes), théoriquement, expérimentalement ou graphiquement, on peut facilement déterminer l'autre groupe et calculer la vitesse. Dans une section transwersale normale d'un écoulcment parallèle, la répartition des pressions est quasi hydrostatique.

Une propriete tant soit peu semblable demenre oraie dans un plan donné pour un écoulement non permanent, visqueux ou non visqueux, pour un coulement compressible, non visqueux et non permanent, et pour un écoulement axisymétrique.

En général, cette proposition n'est pas vraie dans le cas d'un écoulement à trois dimensions, excepté pour certains écoulements à deux dimensions dans des surfaces courbes, ce qui englobe la plupart des cas pratiques.

D'autres propriétés de l'écoulement plan visqueux sont étudiées.
\end{abstract}

\begin{abstract}
Isoclines are lines of equal inclination of the velocity vector. Isovels are lines of equal velocity. Isopiestic lines have equal piezometric head.

In an inviscid incompressible fluid in steady potential flow in a plane the isoclines are normal to the isopiestic lines.

This property holds in rotational flow in a plane, only here the isopiestic lines are not identical with the isovels. In gases at low velocities the isoclines are normal to the isobars.

This theorem has numerous applications: If one set of lines (isoclines or isopiestic lines) is known by theory, experiment or graphically, the other set may easily be determined and the velocity computed. In a normal cross. section of a parallel flow the distribution of pressures is quasi-hydrostatic.

A somewhat similar property holds in a plane for unsteady inviscid or viscous flow, unsteady inviscid compressible flow, and axially symmetrical flow.

In general it is not true in trce-dimensional flow, except for certain two-dimensional flows in curved surfaces, which include most practical cases.

Some other properties of viscous plane flow are investigated.
\end{abstract}

\section{I. - INTRODUCTION}

Considérons le cas simple d'un fluide non visqueux et incompressible en régime permanent et en écoulement irrotationnel ou potentiel. Le vecteur de vitesse $\underline{V}$ dérive d'un potentiel de vitesse scalaire :

$$
\underline{V}=\operatorname{grad} \emptyset
$$

Let us consider the simple case of an inviscid incompressible fluid in steady motion, in irrotational or potential flow. The velocity vector $\underline{V}$ derives from a scalar velocity potentiel 0 :
(1) Exposé fait à la $8^{\mathrm{e}}$ réunion de l'Israel Association for theoretical and applied Mechanies, Haifa, 1958.
(1) Lecture given at the $8 \mathrm{th}$ Meeting of the Israel Association for theoretical and applied Mechanics, Haifa, 1958 . 
Soit $\mathrm{P}$ le potentiel combiné de la pression et des forces de gravité :
If $\mathrm{P}$ is the combined pressure and gravity potential of forces :

$$
\mathrm{P}=g \mathrm{Z}+p / p=g h
$$

avec $h=\mathrm{la}$ hauteur piézométrique ou charge mesurée au moyen de tubes piézométriques: where $h$ is the piezometric head measured by piezometric tubes:

$$
h=Z+p / g e=Z+p / \gamma
$$

( $p=$ pression; $Z=$ altitude au-dessus du niveau de référence; $p=$ densité ou masse spécifique; $y=$ poids unitaire). L'énergie mécanique spécifique $\mathrm{E}$, c'est-à-dire l'énergie par masse unitaire du fluide, est constante partout:
( $p=$ pressure; $Z=$ vertical elevation above datum; $p=$ density or specific mass; $\gamma=$ unit weight). Then the specific mechanical energy E, i.e. per unit mass of fluid, is constant everywhere :

$$
\mathrm{E}=\mathrm{P}+\mathrm{V}^{2} / 2=\mathrm{C}^{\mathrm{te}}
$$

Une « isovel » (isotache, isodrome) est une ligne de vitesse constante. Suivant (1.4), elle est également une ligne sur laquelle $\mathrm{P}$ ou $h$ sont constants; elle est donc en outre une ligne isopièze. Dans le cas des gaz aux faibles vitesses dans la gamme subsonique, les influences de la compressibilité et de la pesanteur sont négligeables. On peut remplacer (1.2) à titre approximatif par:

$$
\mathrm{P} \sim p / \rho
$$

Done $\mathrm{P}=\mathrm{C}^{\text {te }}$ implique que $p=\mathrm{C}^{\text {te }}$, c'est-à-dire soit une isobare.

D'après la thécrie des fonctions, nous savons que si nous définissons une variable complexe par $z=x+i y$, nous pouvons alors considérer toute fonction analytique de celte variable comme étant le potentiel complexe d'un écoulement :

$$
w=f(z)=\varphi+i \psi
$$

puisqu'elle se compose d'une partie réelle $\varphi=\varphi(x, y)$, qui peut représenter un potentiel de vitesse éventuel en écoulement plan, et, d'autre part, d'une partie imaginaire $\psi=\psi(x, y)$, qui représente la fonction de courant correspondante. Le vecteur de vitesse $\mathrm{V}$ est alors normal par rapport aux lignes équipotentielles (lignes $\varphi=\left(\mathrm{C}^{\mathrm{t}}\right)$, et tangente aux lignes de courant (lignes $y=C^{\text {te }}$. I Les équipotentielles et les lignes de courant sont orthogonales les unes aux autres, et constituent un réseau isotherme $\left({ }^{2}\right)$.

(2) Méthode dite « des petits carreaux », exposée par I'. Danel et G. Sauvage De Saint-Marc: : Cours d'hydrodynamique graphique $\gg$, fasc. I, Ecole Nationale Supérieure d'Electrotechnique et d'Hydranlique de Grenoble (1950).
An isovel (isotach, isodrome) is a line of constant velocity. By (1.4) it is also a line of constant $\mathrm{P}$ or $h$, i.e. it is also an isopiestic line. In the case of gases at low subsonic velocities, the effects of compressibility and gravity are negligible. (1.2) may approximately be replaced by :
The theory of functions states : if we define a complex variable by $z=x+i y$, then any analytical function of this variable may be considered as the complex potential of a flow:
(2) Called "Small Squares," see Danfi, P. \& Sauvagr DE SAIN'MARC, G.: "Cours d'hydrodynamique graphique," Fasc. 1, Ecole Vationale Supérieure d'Electrolechnique et d'Hydraulique de Grenoble (1950).

as it consists of a real part $\varphi=\varphi(x, y)$, which may represent a possible velocity potential in plane flow; and of an imaginary part $\psi=\psi(x, y)$ which represents the corresponding stream-function. The velocity vector $\underline{V}$ is then normal to the equipotential lines (of equal $\varphi$ ) and tangent to the streamlines (of equal $\psi$ ). The equipotentials and streamlines are mutually orthogonal and form an isothermal flow-net $\left(^{2}\right)$. 
La première dérivée représente ce que l'on connaît sous le nom de vitesse complexe (3) :
The first derivative represents the so-called complex velocity ${ }^{(3)}$ :

$$
d w / d z=f^{\prime}(z)=u-i v=\mathrm{V} \exp (-i 0)
$$

avec : $0=$ l'inclinaison de la vitesse par rapport à l'axe des $x ; u, v$ étant les composantes cartésiennes de $\underline{\mathrm{V}}$. Nous avons :

$$
\cot \theta=u / v ; \quad \mathrm{V}^{2}=u^{2}+v^{2}
$$

D'après Kirchhoff

Following Kirchhoff

$$
\ln (d w / d z)=\ln \mathrm{V}-i 0
$$

est une autre fonction analytique, d'où il suit que les isoclines (lignes d'égale inclinaison $\theta$ ) sont orthogonales par rapport aux isovels (lignes d'égales valeurs de V), et, par suite de (14), les isoclines sont normales aux lignes isopièzes is another analytical function, hence the isoclines, i.e. lines of equal inclination 0 , are orthogonal to the isovels (equal V), and because of (1.4) the isoclines are normal to the isopiestic lines (fig. 1).

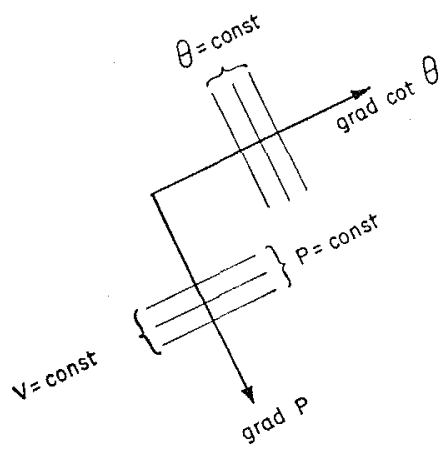

FIG. 1

(fig. 1). Qu'advient-il de cette propriété remarquable en écoulement rotationnel, en mouvement non permanent, dans un fluide compressible, dans un fluide visqueux, en écoulement tri-dimensionnel, en écoulement turbulent?
What happens to this remarkable property in rotational flow, in unsteady motion, in a compressible fluid, in a viscous one, in three dimensions, in turbulent flow?

\section{II. - ECOULEMENT PLAN ROTATIONNEL D'UN FLUIDE INCOMPRESSIBLE NON VISQUEUX EN MOUVEMENT PERMANENT \\ PLANE ROTATIONAL FLOW OF AN INVISCID INCOMPRESSIBLE FLUID IN STEADY MOTION}

Nous obtenons de l'équation de continuité : Equation of continuity gives :

$$
u_{x}+v_{y}=0
$$

(3) Voir Mrlne-Thomson, L. M. : « Theoretical Hydrodynamies 》, Macmillan, London (1955).
(3) See Mrine-Tromson, L. M.: Theoretical Hydrodynamies, Macmillan, London (1955). 
les équations de mouvement d'Euler :

Euler's equations of motion :

$$
\begin{gathered}
u u_{x}+v u_{y}=-\mathrm{P}_{x} \\
u v_{x}+v v_{y}=-\mathrm{P}_{y}
\end{gathered}
$$

En éliminant $u_{x}$ de (2.2) ei $v_{y}$ de (2.3) au moyen de (2.1):

$$
\begin{gathered}
\mathrm{P}_{x}=u v_{y}-v u_{y}=-v^{2}(u / v)_{y}=-v^{2}(\cot \theta)_{y} \\
\mathrm{P}_{y}=v u_{x}-u v_{x}=+v^{2}(u / v)_{x}=+v^{2}(\cot \theta)_{x}
\end{gathered}
$$

En éliminant $v^{2}$ :

Eliminating $v^{2}$ :

$$
\mathrm{P}_{x}(\cot \theta)_{x}+\mathrm{P}_{y}(\cot \theta)_{y}=\operatorname{grad} \mathrm{P} \cdot \operatorname{grad} \cot \theta=0
$$

Le point (.) symbolise la multiplication scalaire. Cette équation démontre l'orthogonalité des lignes isoclines et isopièzes dans un écoulement rotationnel (fig. 2).

En écoulement potentiel, en raison de (1.4), les isoclines sont normales aux isovels.

Dans les gaz à faibles vitesses, en raison de (1.5), les isoclines sont normales aux isobares.

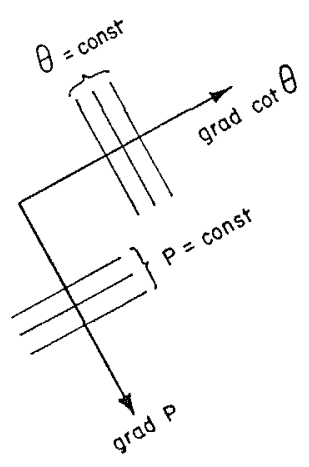

Fig. 2

Cette propriété trouve de nombreuses applications :

a) Si nous connaissons les lignes d'une catégorie (isoclines ou lignes isopièzes) théoriquement, par voie expérimentale ou graphique, nous pouvons aisément déterminer celles de l'autre catégorie; nous pouvons ensuite calculer $v^{2}$ au moyen de (2.4) ou (2.5) (fig. 3).

b) Beaucoup de veines liquides (jets libres, nappes) ont une section transversale plane dans laquelle les filets liquides sont parallèles, avec une courbure tendant à s'annuler, par exemple, la « vena contracta »d'un jet émergeant d'un orifice.
The dot (.) represents scalar multiplication. This equation proves the orthogonality of isoclines and isopiestic lines in rotational flow. (Fig. 2)

In potential flow, because of (1.4), the isoclines are normal to the isovels.

In gases at low velocities, because of (1.5), the isoclines are normal to the isobars.

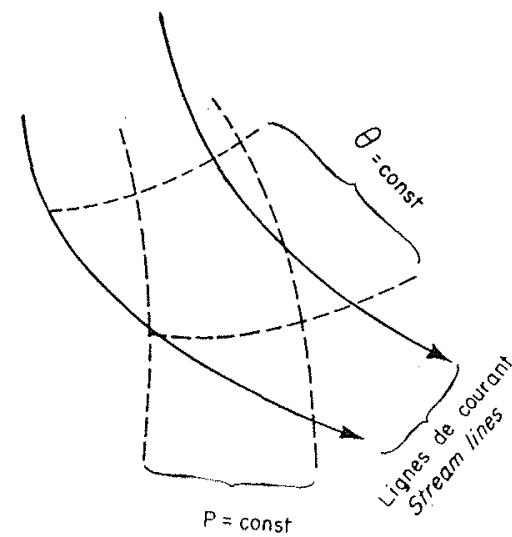

Fic. 3

This property has many applications :

a) If one set of lines" (isoclines, or isopiestic lines) is known by theory, experiment or graphically, the other set may easily be determined. Then $v^{2}$ may be computed by (2.4) or (2.5). (Fig. 3).

b) Many stream tubes (free jets, nappes) have often a plane cross-section where the streamlines are parallel with a vanishing curvature, e.g. vena contracta of a jet issuing from an orifice. In such a normal cross-section $\theta$ is constant, hence $\mathrm{P}$ is constant too by (2.4), (2.5). This reminds us of a similar result obtained in hydrostatics. Hence the rule : 
Dans une section transversale normale telle que celle-ci, $\theta$ est constant, d'où $P$ est constant également d'après (2.4), (2.5). Ceci nous rappelle un résultat similaire obtenu en hydrostatique. D'où nous tirons la loi :

La répartition des pressions dans une section transversale normale à une veine liquide à filets parallèles est quasi hydrostatiquze.

«Quasi », parce que, en hydrostatique, $\mathrm{P}=\mathrm{C}^{\text {te }}$ partout, tandis qu'ici $\mathrm{P}=\mathrm{C}^{\text {te }}$ seulement dans une section normale, et la constante peut varier de section à section.

c) Pour toute paroi solide d'équation $f(x, y)=0$, la ligne de courant est tangente à la paroi : la paroi est une ligne de courant. A la paroi :
The distribution of pressures in a cross-section normal to a parallel stream-tube is quasi-hydrostatic.

Quasi - because in hydrostatics $\mathrm{P}=$ const eve. rywhere, whereas here $\mathrm{P}=$ const only in one normal section, and the constant may be different from section to section.

c) At any solid wall of the equation $f(x, y)=0$ the stream-line is tangent to the wall : the wall is a streamline. At the wall :

et là :

$$
\cot 0=-1 / v=-f_{n} / f_{n}
$$

There :

$\operatorname{grad} \mathrm{P} \cdot \operatorname{grad}\left(f_{y} / f_{x}\right)=0$

\section{III. - ECOULEMENT PLAN NON PERMANENT D'UN FLUIDE INCOMPRESSIBLE NON VISQUEUX \\ PLANE UNSTEADY FLOW OF AN INVISCID INCOMPRESSIBLE FLUID}

(2.2) et (2.3) sont remplacées par :

(2.2) and (2.3) are replaced by :

$$
\begin{aligned}
& u_{t}+u u_{x}+v u_{y}=-\mathrm{P}_{x} \\
& v_{l}+u v_{y}+v v_{y}=-\mathrm{P}_{y}
\end{aligned}
$$

En procédant comme au paragraphe 2, nous obtenons (fig. 4):

Proceeding as in Section 2, we obtain (Fig. 4) :

$$
\left(\mathrm{P}_{p}+u_{t}\right)(\cot \theta)_{x}+\left(\mathrm{P}_{y}+v_{t}\right)(\cot \theta)_{y}=\left(\operatorname{grad} \mathrm{P}+\underline{\mathrm{V}}_{t}\right) \cdot \operatorname{grad} \cot \theta=0
$$

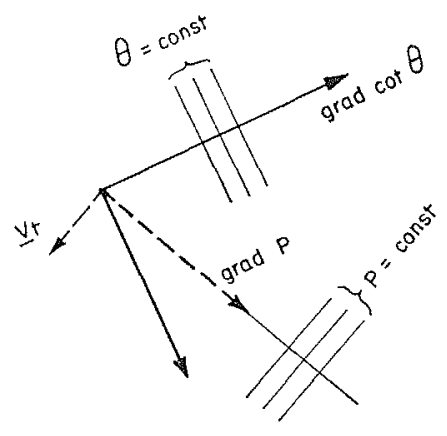

FIG. 4

Dans un jet parallèle :

In parallel jet :

$$
\operatorname{grad} P=-V_{t}
$$




\section{IV. - ECOULEMENT PLAN NON PERMANENT D'UN FLUIDE VISQUEUX INCOMPRESSIBLE \\ PLANE UNSTEADY FLOW OF A VISCOUS INCOMPRESSIBLE FLUID}

(2.2) et (2.3) sont remplacées par les équations de Navier-Stokes :
(2.2) and (2.3) are replaced by the Navier-Stokes equations :

$$
\begin{aligned}
& u u_{t}+u u_{x}+v u_{\eta !}=-\mathbf{P}_{x}+\nu \nabla^{2} u \\
& v_{t}+u v_{x}+v v_{y /}=-\mathrm{P}_{y}+\nu \nabla^{2} v
\end{aligned}
$$

$y=$ viscosité cinématique. En procédant comme au paragraphe 3 , nous obtenons (fig. 5) : $\nu=$ kinematic viscosity. Proceeding as in seclion 3, we obtain (Fig. 5) :

$$
\left(\mathrm{P}_{y}+u_{t}-v \nabla^{2} u\right)(\cot \theta)_{x}+\left(\mathrm{P}_{y}+v_{t}-\nu \nabla^{2} v\right)(\cot \theta)=\left(\operatorname{grad} \mathrm{P}+\underline{\mathrm{V}}_{t}+v \operatorname{rot} \operatorname{rot} \underline{\mathrm{V}} .\right) \operatorname{grad} \cot \theta=0
$$

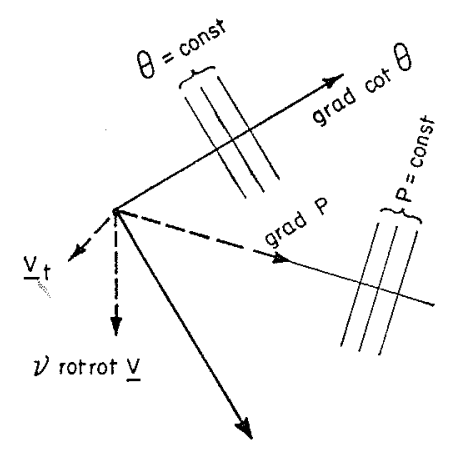

FIG. 5

comme en écoulement incompressible :

as in incompressible flow :

$$
\operatorname{rot} \operatorname{rot} \underline{Y}=-\nabla^{2} \underline{V}
$$

Dans un jet parallèle :

In a parallel jet :

$$
\text { grad } \mathrm{P}=-\underline{V}_{t}-v \operatorname{rot} \operatorname{rot} \underline{\mathrm{V}}
$$

Dans le cas d'un écoulement visqueux en contact avec une paroi plane solide, nous pouvons prendre l'axe des $y$ normal à la paroi et l'axe des $x$ le long de la paroi. Dans ce cas, la paroi est une isocline et le long de celle-ci, (4.5) est valable. Comme, en n'importe quel point de la paroi, $\underline{V}=0$, il en découle que $u, v, \underline{V}_{t}, u_{t}, v_{t}$, $u_{x}, u_{x x}=0$ aussi, à la paroi. Comme, d'après (4.4) également, - rot rot $\underline{V}$ a les composantes $\nabla^{2} u$, $\nabla^{2} v:$

$$
\mathrm{P}_{x}=\text { v. } u_{y y}
$$

d'où la loi :

Si le profil de vitesse est convexe à la paroi $\left(u_{y y}<0\right), \mathrm{P}_{x}<0$, la hauteur piézométrique dé-
In the case of riscous flow in contact with a solid plane wall, we may choose the $y$-axis normal to the wall, the $x$-axis along the wall. In this case the wall is an isocline and along it (4.5) is valid. As at any point of the wall $\underline{V}=0$, hence $u, v, \underline{V}_{t}, u_{t}, v_{t}, u_{x}, u_{x x}=0$ too at the wall. As also by $(4.4)$ - rot rot $\underline{V}$ has the components $\nabla^{2} u, \nabla^{2} v$ : 
croît dans le sens de l'écoulement. Mais si le profil de vitesse est concave à la paroi $\left(u_{m y}>0\right)$, $\mathrm{P}_{w}>0$, la hauteur piézométrique croît dans le sens de l'écoulement et celui-ci est instable $\left(^{(}\right)$. ses in the direction of flow. But it the velocity profile is concave at the wall $\left(u_{y y}>0\right), \mathrm{P}_{x}>0$ the piezometric head increases in the direction of flow and the flow is unstable $\left.{ }^{4}\right)$.

\section{V. - ECOULEMENT PLAN NON PERMANENT D'UN FLUIDE NON VISQUEUX ET COMPRESSIBLE \\ PLANE UNSTEADY FLOW OF AN INVISCID COMPRESSIBLE FLUID}

(3.1) et (3.2) restent valables, mais $P$ n'est pas déterminé par (1.2), mais par :
(3.1) and (3.2) remain valid, only $P$ is not defined by (1.2), but by :

$$
\mathrm{P}=g \mathrm{Z}+\int(d p / \rho)
$$

Nous avons:

We have :

$$
\operatorname{grad} \mathrm{P}=-g \cdot \underline{1_{z}}+(\operatorname{grad} p) / ?
$$

$1_{z}$ est un vecteur unitaire vertical dirigé vers $1_{z}$ - is a vertical unit vector directed upwards. le haut. (2.1) est remplacé par :

(2.1) is replaced by:

$$
(\rho u)_{y}+(\rho v)_{y}+\rho_{i}=0
$$

ou :

or :

$$
u_{x}+v_{y}=-(\underline{\mathrm{V}} \cdot \operatorname{grad} \ln \varphi)-(\ln \rho)_{t}
$$

En procédant comme ci-dessus, nous obte- Proceeding as above, we obtain : nons :

$$
\begin{gathered}
{\left[\mathrm{P}_{x}+u_{t}-u(\underline{\mathrm{V}} \cdot \operatorname{grad} \ln \rho)-u(\ln \rho)_{t}\right](\cot \theta)_{x}+\left[\mathrm{P}_{y}+v_{t}-v(\underline{\mathrm{V}} \cdot \operatorname{grad} \ln \rho)-v(\ln \rho)_{t}\right] \cdot(\cot 0)_{y}} \\
\left.=\left\{\operatorname{grad} \mathrm{P}+\underline{\mathrm{V}}_{t}-\underline{\mathrm{V}}[\underline{\mathrm{V}} \cdot \operatorname{grad} \ln \rho)+(\ln \rho)_{t}\right]\right\} \cdot \operatorname{grad} \cot \theta=0
\end{gathered}
$$

Dans un jet parallèle :

In a parallel jet :

$$
\operatorname{grad} \mathrm{P}=-\underline{\mathrm{V}}_{t}+\underline{\mathrm{V}}\left[(\underline{\mathrm{V}} \cdot \operatorname{grad} \ln \rho)+(\ln \rho)_{t}\right]
$$

En écoulement permanent (fig. 6) :

In steady flow (Fig. 6):

$$
\text { grad } \mathrm{P}=b \cdot \underline{\mathrm{V}}
$$

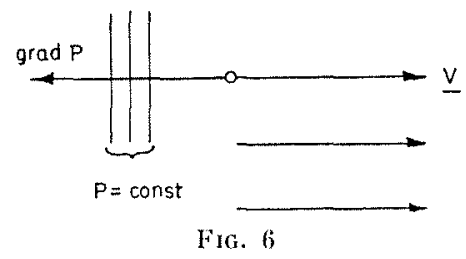

(4) Ceci correspond au clitère de l'instabilité de l'écoulement de Rayleigh-Tollmien : le profil concave ne peut crô̂tre jusqu'à l'infini, de sorte qu'il doit s'infléchir quelque part et il devient convexe. L'existence d'un point d'inflexion est un critère d'instabilité suffisant. Voil Schuchtina, H. : «Grenzschicht-Theorie 》, Braun, Karlsiuhe (1951); p. 59; LrN, C. C. : "The theory of hydrodynamic stability », Camb. Univ. Press (1955).
(4) This corresponds to the Rayleigh-Tollmien criterion of instability of flow: the concave profile cannot grow to infinity, so it has to turn somewhere and becomes convex. The existence of an inflexion point is a sufficient criterion of instability. See Schrichting, H.: Grenzschicht-Theorie, Braun, Karlsruhe (1951), p. 59; LiN, C. C.: The theory of hydrodynamic stability, Camb. Univ. Press (1955). 


\section{VI. - ECOULEMENT AXI-SYMÉTRIQUE D'UN FLUIDE NON VISQUEUX ET INCOMPRESSIBLE}

\section{AXIALLY-SYMMETRICAL FLOW OF AN INVISCID INCOMPRESSIBLE FLUID}

Equation de continuité :

Equation of continuity :

$$
\left(r^{\prime} u\right)_{r}+(r v)_{y}=0
$$

Equations de mouvement d'Euler en coordonnées ${ }^{(5)}$ cylindriques :

Euler's equations of motion in cylindrical coordinates (s):

$$
\begin{gathered}
u_{t}+u u_{r}+v u_{y}=-\mathrm{P}_{r} \\
v_{t}+-u v_{r}+v v_{y}=-\mathrm{P}_{y} \\
\cot 0=u / v
\end{gathered}
$$

$u=$ composante radiate (direction $r) ; \quad v=$ composante axiale (direction $y$ ).

En éliminant $u_{r}$ de (6.2) et $v_{y}$ de $(6.3)$ au moyen de (6.1): $u=$ radial component (direction $r$ ); $v=$ axial component (direction $y$ ).

Eliminating $u_{r}$ from (6.2) and $v_{y}$ from (6.3) by means of $(6.1)$ :

$$
\begin{aligned}
& \mathrm{P}_{r}+u_{t}-u^{2} / r=-v^{2}(\cot \theta)_{y} \\
& \mathrm{P}_{y}+v_{t}-u v / r=+v^{2}(\cot \theta)_{r}
\end{aligned}
$$

En élininant $v^{2}$ :

Eliminating $b^{2}$ :

$$
\left(\mathbf{P}_{r}+u_{t}-u \cdot u / r\right)(\cot \theta)_{r}+\left(\mathrm{P}_{y}+v_{t}-v \cdot u / r\right)(\cot \theta)_{y}=\left(\operatorname{grad} \mathrm{P}+\mathrm{V}_{t}-\underline{\mathrm{V}} \cdot u / r\right) \cdot \operatorname{grad} \cot \theta=0
$$

Ceci est montré sur la figure 7 pour l'écoule- In steady flow this is shown in Fig. 7 : ment permanent :

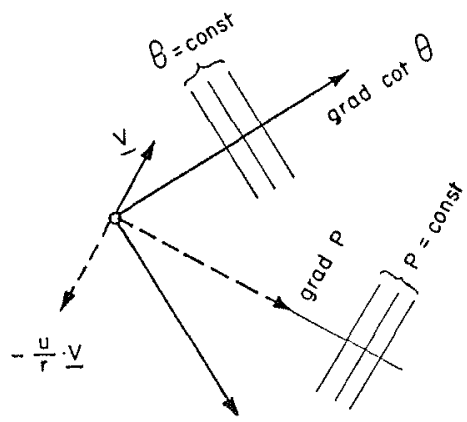

FIG. 7

Ici $\theta=\mathrm{C}^{\text {te }}$ signifie que nous avons un écoulement parallèle dans un plan diamétral, ce plan tournant autour d'un axe.

Dans un tel écoulement :
Here $\theta=$ const means parallel flow in a diametral plane, the plane being rotated round an axis.

In such a flow :

$$
\operatorname{grad} \mathrm{P}=\underline{\mathrm{V}}_{t}-\underline{\mathrm{V}} \cdot u / r
$$

(5) Sans le terme azimutal, à cause de la symétrie axiale.
(5) Without the azimuthal term, because of the axial symmetry. 


\section{VII. - ECOULEMENT CURVILIGNE A TROIS DIMENSIONS D'UN FLUIDE VISQUEUX ET INCOMPRESSIBLE \\ THREE-DIMENSIONAL CURVILINEAR FLOW OF A VISCOUS INCOMPRESSIBLE FLUID}

L'écoulement visqueux d'un fluide incompressible obéit à l'équation de continuité :
Viscous flow of an incompressible fluid obeys the equation of continuity :

$$
\operatorname{div} \underline{V}=0
$$

et aux équations de mouvement de NavierStokes qui donnent le vecteur d'accélération $a$ : and the Navier-Stokes equations of motion which give the acceleration vector $a$ :

$$
\underline{a}=\underline{\mathrm{V}}_{t}-\underline{\mathrm{V}} \times \operatorname{rot} \underline{\mathrm{V}}+\operatorname{grad}\left(\mathrm{V}^{2} / 2\right)=-\operatorname{grad} \mathrm{P}-\nu \operatorname{rot} \operatorname{rot} \underline{\mathrm{V}}
$$

$\times$ : symbole de multiplication vectorielle.

Considérons trois familles de surfaces réciproquement orthogonales dans un système de coordonnées cartésicnnes $\mathrm{X}, \mathrm{Y}, \mathrm{Z}$ :
$(X)$ - symbol of vectorial multiplication.

Let us consider in a cartesian system of coordinates $\mathrm{X}, Z, Y$, three families of mutually orthogonal surfaces :

$$
f_{1}(\mathrm{X}, \mathrm{Y}, Z)=x ; \quad f_{2}(\mathrm{X}, \mathrm{Y}, \mathrm{Z})=y ; \quad f_{3}(\mathrm{X}, \mathrm{Y}, Z)=z
$$

En général, il n'y a qu'une seule surface de chaque famille qui passe par un point donné $\mathrm{M}(\mathrm{X}, \mathrm{Y}, \mathrm{Z})$, que l'on peul également définir par les trois paramètres ou coordonnées générates ou curvilignes $x, y, z\left(^{(6)}\right.$.

La longueur de la normale limitée par les surfaces $x$ et $x+d x$ est donnée par :
In general only one surface of each family passes through a given point $\mathrm{M}(\mathrm{X}, \mathrm{Y}, \mathrm{Z})$, which may also be defined by the three general or curvilinear parameters or coordinates $x, y, z{ }^{(i)}$.

The length of the normal intercepted between the surfaces $x$ and $x+d x$ is given by :

$$
d s_{1}=h_{1} d x ; \quad 1 / h_{1}^{2}=x_{\mathrm{X}}^{2}+x_{\mathrm{Y}}^{2}+x_{\mathrm{Z}}^{2}
$$

Les indices inférieurs représentent des dérivées partielles. De mème façon, la distance normale entre les surfaces $y, y+d y$ est $h_{2} d y$, et celle entre les surfaces $z, z+d z$ est $h_{3} d z$.

Soit $u, v, w$ les composantes de la vitesse $\underline{\mathrm{V}}$ dans les trois directions de $x, y, z$ augmentant (appelées également les lignes $x, y, z$ ).

L'équation de continuité (7.1) devient:
The lower letter indices represent partial derivatives. Similarly the normal distance between the surfaces $y, y+d y$ is $h_{2} d y$, and between the surfaces $z, z+d z$ it is $h_{3} d z$.

Let $u, v, w$ be the components of the velocity $\underline{V}$ in the three directions of $x, y, z$ increasing (called also the $x, y, z$-lines).

Equation of continuity (7.1) becomes:

$$
h_{1} h_{2} h_{3} \operatorname{div} \underline{\mathrm{V}}=\left(h_{2} h_{3} u\right)_{x}+\left(h_{3} h_{1} v\right)_{y}+\left(h_{1} h_{2} w\right)_{x}=0
$$

(6) Voir Love (A. E. H.) : \& A treatise on the mathematical theory of elasticity », Camb. Univ. Press (1927), page 51. Nous écrivons $h_{i}$ comme on le fait aujourd'hui, qui correspond au $1 / h_{3}$ de Love; voir par exemple MILNEThomson, l.c., p. 57-60; Stratton (J. A.) : « Electromagnetic Theory 》, McGraw-Hill, New York (1941), p. 47-59; OltendorfF (F.) : " Die Welt der Vectoren, Springer, Vienne (1950); BridjoulN (L.) : « Les tenseurs en mécanique et en élasticité », Dover, New York (1946). Les $h_{i}^{2}$ sont équivalents aux composantes $g_{i i}$ du tenseur métrique de Riemann.
(6) See Love, A. E. H.: "A treatise on the mathematical theory of elasticity," Camb. Univ. Press (1927), p. 51. We write $h_{i}$ for Love's $1 / h_{i}$ following modern usage, see e. g. Milne-Thomson, l. e., p. 57-60; Stratron, J.A.: "Electromagnetic theory," McGraw-Hill, New York (1941), p. 47-59; OLLENDORFF, F.: "Die Welt der Verktoren," Springer, Wien (1950); Brillouin, L.: "Les tenseurs en mécanique et en élasticité," Dover, New York (1946). The $h_{i}{ }^{2}$ are equivalent to the components $g_{i t}$ of the Riemannian metric tensor. 
En écoulement à deux dimensiors, c'est-à-dire écoulement dans la surface courbe $z=\mathrm{C}^{\text {te }}$, nous avons $w=0$ et $\partial / \partial z=0$.

Alors (7.5) devient :

$$
\left(h_{2} h_{3} u\right)_{x}+\left(h_{3} h_{1} v\right)_{y}=h_{2} h_{3} u_{x}+h_{3} h_{1} v_{y}+\left(h_{2} h_{3}\right)_{x} u+\left(h_{3} h_{1}\right)_{y} v=0
$$

Le vecteur gradient $P$ a les composantes $\mathrm{P}_{x} / h_{1} ; \mathrm{P}_{y} / h_{2} ; 0$

$\underline{V}_{t}$ a les composantes $u_{t} ; v_{t} ; 0$

Le vecteur $\operatorname{grad}\left(\mathrm{V}^{2} / 2\right)=\operatorname{grad}\left[\left(u^{2}+v^{2}\right) / 2\right]$ a les composantes:

$$
\left(u u_{x}+v v_{v}\right) / h_{1} ; \quad\left(u u_{y}+v v_{y}\right) / h_{2} ; \quad 0
$$

Le vecteur rot $\underline{V}$ est défini par :

$$
h_{1} h_{2} h_{3} \operatorname{rot} \mathrm{V}=\left|\begin{array}{lll}
\underline{h_{1}} 1_{x} & \underline{h_{2}} 1_{y} & \underline{h_{32} 1_{z}} \\
\partial / \partial x & \partial / \partial y & \partial / \partial z \\
h_{1} u & h_{1} v & h_{1} w
\end{array}\right|
$$

et a les composantes :

$$
0 ; \quad 0 ; \quad\left[\left(h_{2} v\right)_{x}-\left(h_{1} u\right)_{y}\right] / h_{1} h_{2}
$$

Le vecteur :

$$
\underline{\mathrm{V}} \times \operatorname{rot} \underline{\mathrm{V}}=\left|\begin{array}{lll}
\underline{1_{x}} & \underline{1_{y}} & \underline{1_{\underline{z}}} \\
u & v & w \\
0 & 0 & \left.\left[\left(h_{2}\right) v\right)_{x}-\left(h_{1} u\right)_{y}\right] / h_{1} h_{2}
\end{array}\right|
$$

a les composantes :

$$
\left[\left(h_{2} v\right)_{x}-\left(h_{1} u\right)_{y}\right] v / h_{1} h_{2}
$$

Le vecteur rot rot $\underline{V}$ tel qu'il est défini par :

has the components :

$$
-\left[\left(h_{2} v\right)_{x}-\left(h_{1} u\right)_{y}\right] u / h_{1} h_{2} ; \quad 0
$$

The vector rot rot $\underline{V}$ as defined by :

$$
\begin{array}{llll}
h_{1} h_{2} h_{3} \operatorname{rot} \operatorname{rot} \underline{\mathrm{V}}= & \underline{h_{1} 1_{x}} & \underline{h_{2}} 1_{y} & \underline{h_{3} 1_{z}} \\
\partial / \partial x & \partial / \partial y & \partial / \partial z \\
0 & 0 & {\left[\left(h_{2} v\right)_{a}-\left(h_{1} u\right)_{y}\right] / h_{1} h_{2}}
\end{array}
$$

a les composantes :

has the components:

$$
\left\{\left[\left(h_{2} v\right)_{x}-\left(h_{1} u\right)_{y}\right] / h_{1} h_{2}\right\}_{y} / h_{2} h_{3}
$$$$
-\left\{\left[\left(h_{2} v\right)_{x}-\left(h_{1} u\right)_{y}\right] / h_{1} h_{2} \dot{\gamma}_{a} / h_{1} h_{3}\right.
$$

En introduisant ces expressions dans (7.2) :

Introducing these expressions into (7-2) :

$$
\begin{aligned}
& a_{1}=u_{t}-\left[\left(h_{2} v\right)_{z}-\left(h_{1} u\right)_{y}\right] v / h_{1} h_{2}+\left(u u_{x}+v v_{x}\right) / h_{1}=-P_{x} / h_{1}-\left\{\left[\left(h_{2} v\right)_{x}-\left(h_{1} u\right)_{y}\right] / h_{1} h_{2}\right\}_{y} \nu / h_{2} h_{z} \\
& a_{2}=v_{t}+\left[\left(h_{2} v\right)_{x}-\left(h_{1} u\right)_{y}\right] u u_{i}^{\prime} h_{1} h_{2}+\left(u u_{y}+v v_{y}\right) / h_{2}=-\mathrm{P}_{y} / h_{1}+\left\{\left[\left(h_{2} v\right)_{x}-\left(h_{1} u\right)_{y}\right] / h_{1} h_{2}\right\}_{x} \nu / h_{1} h_{3}
\end{aligned}
$$


En mettant sous une autre forme, après avoir éliminé $u_{x}$ de (7.16) et $v_{y}$ de (7.17) au moyen de (7.6), nous obtenons :
Re-arranging, after elimination of $u_{x}$ from (7.16) and of $v_{y}$ from (7.17) by means of (7.6), we get :

$$
\begin{gathered}
\mathrm{P}_{x} / h_{1}+u_{t}+v(\operatorname{rot} \operatorname{rot} \underline{V})_{1}=(u / v)_{y} v^{2} / h_{2}+u v .\left(\ln h_{3}\right)_{y} / h_{2}+u^{2} \cdot\left(\ln h_{3}\right)_{x} / h_{1}+\mathrm{V}^{2}\left(\ln h_{\underline{2}}\right)_{x} / h_{1} \\
\mathrm{P}_{y} / h_{2}+v_{t}+v(\operatorname{rot} \cot \underline{\mathrm{V}})_{2}=-(u / v)_{x} v^{2} / h_{1}+v^{2}\left(\ln h_{3}\right)_{y} / h_{2}+u v .\left(\ln h_{3}\right)_{x} / h_{1}+\mathrm{V}^{2}\left(\ln h_{1}\right)_{y} / h_{1}
\end{gathered}
$$

En multipliant (7.18) par $(u / v)_{x} / h_{1}$ et (7.19) par $(u / v)_{y} / h_{2}$, et en ajoutant :

Multiplying (7.18) by $(u / v)_{\alpha} / h_{1}$ and by $(u / v)_{y} / h_{\mathfrak{s}}$, and adding :

$$
\begin{aligned}
& {\left[\operatorname{grad} \mathrm{P}+\underline{\mathrm{V}}_{t}+v \operatorname{rot} \operatorname{rot} \underline{\mathrm{V}}+\underline{\mathrm{V}}\left(\underline{\mathrm{V}} \cdot \operatorname{grad} \ln h_{3}\right)\right] \cdot \operatorname{grad}(u / v)} \\
& =\mathrm{V}^{2}\left[\left(\ln h_{2}\right)_{w} / h_{1} \cdot(u / v)_{t \cdot} / h_{1}+\left(\ln h_{1}\right)_{y} / h_{2} \cdot(u / v)_{y} / h_{2}\right]
\end{aligned}
$$

a) Quand $h_{1}=h_{2} \cdot f(x)$

a) When,$h_{1}=h_{2} \cdot f(x)$

$$
\left[\operatorname{grad} \mathrm{P}+\underline{\mathrm{V}}_{t}+v \operatorname{rot} \operatorname{rot} \underline{\mathrm{V}}+\underline{\mathrm{V}}\left(\underline{\mathrm{V}} \cdot \operatorname{grad} \ln h_{3}\right)--\mathrm{V}^{2} \operatorname{grad} l n h_{2}\right] \cdot \operatorname{grad}(u / v)=0
$$

b) Quand $h_{1}=h_{2} f(y)$ :

b) When $h_{1}=h_{2} \cdot f(y)$.

$$
\left[\operatorname{grad} \mathrm{P}+\underline{\mathrm{V}}_{t}+\nu \operatorname{rot} \operatorname{rot} \underline{\mathrm{V}}+\underline{\mathrm{V}}\left(\underline{\mathrm{V}} \cdot \operatorname{grad} \ln h_{3}\right)-\mathrm{V}^{2} \operatorname{grad} \ln h_{1}\right] \cdot \operatorname{grad}(u / v)=0
$$

c) Quand $h_{2 i}=0 ; h_{1 y}=0$

c) When $h_{2 x}=0 ; h_{1 y}=0$

$$
\left[\operatorname{grad} \mathbf{P}+\underline{\mathrm{V}}_{t}+v \operatorname{rot} \operatorname{rot} \underline{\mathrm{V}}+\underline{\mathrm{V}}\left(\mathrm{V} \cdot \operatorname{grad} \ln h_{3}\right)\right] \cdot \operatorname{grad}(u / p)=0
$$

d) Quand $h_{1}=h_{2}=h_{3}=1$ (coordonnées cartésiennes, nous obtenons la formule (4.3) pour écoulement plan simple.

\section{EXFMPLES :}

1) Coordonnées cylindriques: d) When $h_{1}=h_{2}=h_{3}=1$ (cartesian coordinates) we obtain formula (4.3) for simple plane flow.

\section{Examples :}

1) Cylindrical coordinates :

$$
\begin{array}{ll}
x=r ; & h_{1}=1 \\
y=y ; & h_{2}=1 \\
z=0 ; & h_{3}=r
\end{array}
$$

Ici $h_{1}=h_{2}$, d'où, suivant (7.22), parce que $\left(\ln h_{3}\right)=1 / r$ :

Here $h_{1}=h_{2}$, hence by $(7.22)$ as $\left(\ln h_{3}\right)=1 / r:$

$$
\left(\operatorname{grad} \mathrm{P}+\underline{\mathrm{V}}_{t}+v \operatorname{rot} \operatorname{rot} \underline{\mathrm{V}}-\underline{\mathrm{V}} \cdot u / r\right) \operatorname{grad}(u / v)=0
$$

Ce qui est exactement la formule (6.7).

2) Coordonnées paraboliques :

$$
h_{1}=h_{2}=\sqrt{x^{2}+y^{2}} ; \quad h_{3}=x y
$$

$\mathrm{Si}$, dans une zone $u / v=\mathrm{C}^{\text {te }}$, le jet n'est pas parallèle, mais est caractérisé par une inclinaison égale par rapport anx courbes des $x$, nous avons alors :

Dans le cas a):
This is exactly formula (6.7).

2) Parabolic coordinates :

If in a zone $u / v=$ const, the jet is not parallel, but is characterised by equal inclination to the curved $x$-lines; we have then :

In case $a)$ :

$$
\operatorname{grad} \mathrm{P}=-\underline{V}_{t}-v \operatorname{rot} \operatorname{rot} \underline{\mathrm{V}}-\underline{\mathrm{V}}\left(\underline{\mathrm{V}} \cdot \operatorname{grad} \ln h_{3}\right)+\mathrm{V}^{2} \operatorname{grad} \ln h_{\mathbf{2}}
$$


Dans le cas $b$ ) :

In case $b$ ) :

$$
\operatorname{grad} \mathrm{P}=-\underline{\mathrm{V}}_{t}-v \operatorname{rot} \operatorname{rot} \underline{\mathrm{V}}-\underline{\mathrm{V}}\left(\underline{\mathrm{V}} \cdot \operatorname{grad} \ln h_{3}\right)+\mathrm{V}^{2} \operatorname{grad} \ln h_{1}
$$

Dans le cas $c$ ):

In case $c$ ) :

$$
\operatorname{grad} \mathrm{P}=-\underline{\mathrm{V}}_{t}-v \operatorname{rot} \operatorname{rot} \underline{\mathrm{V}}-\underline{\mathrm{V}}\left(\underline{\mathrm{V}} \cdot \operatorname{grad} \ln h_{s}\right)
$$

Dans le cas général, il n'existe pas de relation d'orthogonalité dans les surfaces courbes, excepté dans les cas $a$ ), $b$ ), $c$ ).
In the general case there exists no orthogonality relationship in curved surfaces, except in the cases $a$ ), $b$ ), $c$ ).

\section{VIII. - SUR D'AUTRES PROPRIETTÉS D'UN ÉCOULEMENT PLAN VISQUEUX PERMANENT \\ ON FURTHER PROPERTIES OF PLANE STEADY VISCOUS FLOW}

Les équations de Navier-Stokes (4.1), (4.2) sont :

$$
\begin{aligned}
& u u u_{x}+v u_{y y}=-\mathrm{P}_{x}+\nu\left(u_{x x y}+u_{y y y}\right) \\
& u v_{x}+v v_{y j}=-\mathrm{P}_{y y}+v\left(v_{x x}+v_{y y y}\right)
\end{aligned}
$$

La continuité donne :

Continuity gives :

$$
u_{x}+v_{\nu}=0
$$

En éliminant $u_{x}$ de (8.1) et $v_{y}$ de (8.2) d'après (8.3):

$$
\begin{gathered}
v u_{y}-u v_{y}=v^{2}(\cot \theta)_{y}=-\mathbf{P}_{x y}-v \zeta_{y y} \\
u v_{x}-v u_{x}=-\cdots v^{2}(\cot \theta)_{y}=-\mathrm{P}_{y}+v \zeta_{y x}
\end{gathered}
$$

où la seule composante de vorticité (dans la direction z) est :

where the only component of vorticity (in direction $z$ ) is :

$$
\zeta=v_{x}--u_{y}
$$

En multipliant (8.4) par $(\cot \theta)_{w}$ et (8.5) par $(\cot \theta)_{y}$ et ajoutant :

Multiplying (8.4) by $(\cot 0)_{x}$ and (8.5) by $(\cot \theta)_{y}$ and adding :

$$
\operatorname{grad} P \cdot \operatorname{grad} \cot \theta=-1[\operatorname{grad} \zeta \times \operatorname{grad} \cot \theta]_{3}
$$

où l'indice inférieur 3 représente la composante du produit vectoriel (symbole $X$ ) le long de $Z$.

En ajoutant (8.3) multiplié par $u$, à (8.4), et multiplié par $v$, à (8.5), nous obtenons : where the lower index 3 represents the component of the vectorial product (symbol $X$ ) along $Z$.

Adding (8.3) multiplied by $u$ to (8.4), and multiplied by $v$ to $(8.5)$, we get :

$$
\begin{aligned}
& \left(u^{2}\right)_{x}+(u v)_{y}=-\mathrm{P}_{x}-v \zeta_{y} \\
& (u v)_{x}+\left(v^{2}\right)_{y}=-\mathrm{P}_{y}+v \zeta_{x}
\end{aligned}
$$


En introduisant l'énergie mécanique spécifique de l'écoulement (par rapport à la masse unitaire):

$$
\begin{gathered}
\mathrm{E}=\mathrm{P}+\mathrm{V}^{2} / 2=\mathrm{P}+\left(u^{2}+\nu^{2}\right) / 2 \\
\left(\frac{u^{2}-v^{2}}{2}\right)_{x}+(u v)_{y}=-\mathrm{E}_{x}-v \zeta_{y, l} \\
(u v)_{x}-\left(\frac{u^{2}-v^{2}}{2}\right)_{y}=-\mathrm{E}_{u}+v \zeta_{x}
\end{gathered}
$$

ou :

or :

$$
\begin{aligned}
& \left(\frac{u^{2}-v^{2}}{2}+\mathrm{E}\right)_{x}+(u v+v \zeta)_{y}=0 \\
& \left(\frac{u^{2}-v^{2}}{2}+\mathrm{E}\right)_{y}+(u v-v \zeta)_{y}=0
\end{aligned}
$$

En dérivant (8.11) par rapport à $x$ (puis à $y$ ), et (8.12) par rapport à $y$ (puis à $x$ ), en ajoutant et en soustrayant :
Differentiating (8.11) with respect to $x$ (then $y$ ), and (8.12) with respect to $y$ (then $x$ ), adding and subtracting:

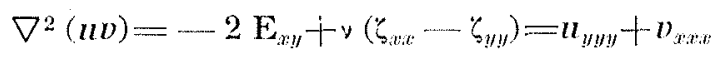

$$
\begin{aligned}
& \nabla^{2} \frac{u^{2}-v^{2}}{2}=\mathrm{E}_{m y}-\mathrm{E}_{x x}-2 \nu_{x u l} \\
& (u b)_{y y}-(u v)_{x y}+\left(u^{2}-v^{2}\right)_{u y}=-v \nabla^{2} \zeta \\
& \left(u^{2}-v^{2}\right)_{x}-\left(u^{2}-v^{2}\right)_{m u}+4(u v)_{w y}=\cdots \nabla^{2} \mathrm{E}
\end{aligned}
$$

(8.8) et (8.9) peuvent également s'écrire:

(8.8) and (8.9) may be written also:

$$
\begin{aligned}
& \left(u^{2}+\mathrm{P}\right)_{x}+(u \nu+v \zeta)_{y}=0 \\
& \left.\left(u^{2}+\mathrm{P}\right)_{y}+(u v)-v \zeta\right)_{x}=0
\end{aligned}
$$

En dérivant (8.19) par rapport à $x$, et (8.20) par rapport à $y$, en ajoutant et en soustrayant :
Differentiating (8.19) with respect to $x$, and (8.20) with respect to $y$, adding and subtracting :

$$
\begin{aligned}
& \left(u^{2}+\mathrm{P}\right)_{x: x}-\left(v^{2}+\mathrm{P}\right)_{y y}+2 v \zeta_{x y}=0 \\
& \left(u^{2}+\mathrm{P}\right)_{x x}+\left(v^{2}+\mathrm{P}\right)_{y y}+2(u v)_{n y}=0
\end{aligned}
$$

